\title{
Domain Walls in the Early Universe and Generation of Matter and Antimatter Domains
}

\author{
A.D. Dolgov ${ }^{1,2, a}$, S.I. Godunov ${ }^{1,2, b}$, and A.S. Rudenko ${ }^{1,3, c}$ \\ ${ }^{1}$ Novosibirsk State University, Novosibirsk, 630090, Russia \\ ${ }^{2}$ Institute for Theoretical and Experimental Physics, Moscow, 117218, Russia \\ ${ }^{3}$ Budker Institute of Nuclear Physics, Novosibirsk, 630090, Russia
}

\begin{abstract}
We present a model where it is possible to generate cosmologically large domains of matter and antimatter separated by cosmologically large distances. Domain walls existed only in the early universe and later they disappeared. So the problem of domain walls in this model does not exist. These features are achieved through a postulated form of interaction between inflaton and a new scalar field.

This scenario inspired a study of the related problem - evolution of the domain wall width in expanding universe. According to classical results there is a region of parameter space where the solutions with constant physical width exist. Numerical study of the problem demonstrates that initial configurations tend to these solutions with time. However, we have found that the wall width can grow exponentially outside of that parameter region.
\end{abstract}

\section{Introduction}

As far as we know, our local cosmological neighborhood is made of baryons, while fraction of antimatter is vanishingly small. Therefore, it looks like the universe is $100 \%$ baryo-asymmetric (at least locally). The Standard Model (SM) cannot explain this asymmetry (BAU). Many quite different extensions of the SM and various scenarios for the BAU generation were suggested in the literature, for a review see e.g. [1-5]. Typically, consideration is restricted to the models where the universe is asymmetric globally. However, it is not excluded that the real universe may be globally symmetric. It may consist of domains of matter and antimatter, and if the domains are sufficiently large and far away, they may escape observational constraints on matter-antimatter annihilation at the domain boundaries. In the simplest version of the scenario the distance to the nearest domain of antimatter should be close to the present day cosmological horizon [6].

Corresponding particle physics models, leading to the universe creation with abundant antimatter domains were suggested and developed in the past. While being more involved, the models of this type also suffer from the inherent problem - a domain wall problem [7]. However, there is a way out of this restriction: domain walls should exist only in the past and should disappear by now, though their

\footnotetext{
ae-mail: dolgov@fe.infn.it

be-mail: sgodunov@itep.ru

ce-mail: a.s.rudenko@inp.nsk.su
} 
effects in the form of matter and antimatter objects would survive to the present day. In [8-11] the class of such a models has been suggested. The main idea behind is a possibility of an unusual symmetry behavior at high temperatures. It is well known that a symmetry, which is broken in vacuum, at high temperatures tends to be restored. But in general, it is also possible for a symmetry to be broken only in a specific range of temperatures. However, if a model is based on the unusual symmetry behavior at high temperatures, then the size of domains will be too small from the cosmological point of view. We suggest the scenario in which this happens during inflationary stage. Domains with different sign of $C P$ disappear by now, so the domain wall problem is absent. However, they appeared during inflation and survived at the baryogenesis epoch, therefore, cosmologically large domains of matter and antimatter could be created. Let us note that the matter-antimatter domains should be separated by at least several megaparsec in terms of the present day scale to avoid excessive matter-antimatter annihilation. On the other hand, the distance should not be too large, otherwise the scenario would lead to too large angular fluctuations of CMB [6].

The scenario described above inspired the consideration of the related problem: is it possible for the domain wall width to grow fast enough in the inflationary universe? The evolution of the domain walls in de Sitter space-time was considered by Basu and Vilenkin [12], where it was argued that the width of the wall tends to the stationary solution for $C \equiv \lambda \eta^{2} / H^{2}>2$, where $H$ is the Hubble parameter, which was assumed to be constant, $\eta$ is the vacuum expectation value of the Higgs-like field which induced the spontaneous symmetry breaking, and $\lambda$ is the coupling constant in the doublewell potential, see (14). If $C \gg 2$, the width of the domain wall is close to its flat space-time value which is microscopically small.

On the other hand, if $C<2$, it is not excluded that the width of the domain wall may be astronomically large. We show that this is indeed the case. In [12] only the stationary problem was considered, when the shape of the domain wall was a function of a single variable, the physical distance. In the paper [12] it was found numerically that the stationary solution exists only if $C>2$.

In what follows we lift the assumption of the stationarity and consider the general plane solution being a function of both variables: the distance from the wall and time. It allows us to see how the solution approaches the stationary one and, in particular, what happens with initial configurations if $C \leq 2$, when the stationary solution does not exist. The related problems were considered in [13].

This talk is based on papers [14] (written in collaboration with I.I. Tkachev) and [15].

\section{Generation of matter and antimatter domains}

\subsection{Evolution of scalar fields during inflation}

The difference between matter and antimatter is to be generated by pseudoscalar field $\chi$ which interacts with inflaton field $\Phi$. We assume the following Lagrangian:

$$
\begin{aligned}
L & =L_{\Phi}+L_{\chi}+L_{i n t}, \\
L_{\Phi}=\frac{1}{2}(\partial \Phi)^{2}-\frac{1}{2} M^{2} \Phi^{2}, \quad L_{\chi} & =\frac{1}{2}(\partial \chi)^{2}-\frac{1}{2} m^{2} \chi^{2}-\frac{1}{4} \lambda_{\chi} \chi^{4}, \quad L_{i n t}=\mu^{2} \chi^{2} V(\Phi),
\end{aligned}
$$

where $M, m, \lambda_{\chi}, \mu$ are constant parameters. Let us note that quadratic shape of the inflaton potential is not crucial here.

We choose $V(\Phi)$ as Gaussian function:

$$
V(\Phi)=\exp \left[-\frac{\left(\Phi-\Phi_{0}\right)^{2}}{2 \Phi_{1}^{2}}\right],
$$


where parameters $\Phi_{0}$ and $\Phi_{1}$ have dimension of mass.

The equations of motion have the form:

$$
\begin{gathered}
\ddot{\Phi}+3 H \dot{\Phi}+M^{2} \Phi+\mu^{2} \chi^{2} \frac{\Phi-\Phi_{0}}{\Phi_{1}^{2}} V(\Phi)=0, \\
\ddot{\chi}+3 H \dot{\chi}+m^{2} \chi+\lambda_{\chi} \chi^{3}-2 \mu^{2} \chi V(\Phi)=0,
\end{gathered}
$$

where $H=\dot{a} / a$ is the Hubble parameter, $a(t)$ is the cosmological scale factor which enters into the FLRW metric as

$$
d s^{2}=d t^{2}-a^{2}(t) d \mathbf{x}^{2} .
$$

It is assumed here that fields $\Phi$ and $\chi$ depend only on time, $\Phi=\Phi(t)$ and $\chi=\chi(t)$.

The Hubble parameter is expressed through the energy density $\rho$ as

$$
H=\sqrt{\frac{8 \pi \rho}{3 m_{P l}^{2}}}=\sqrt{\frac{8 \pi}{3 m_{P l}^{2}}\left(\frac{\dot{\Phi}^{2}}{2}+\frac{M^{2} \Phi^{2}}{2}+\frac{\dot{\chi}^{2}}{2}+\frac{m^{2} \chi^{2}}{2}+\frac{\lambda_{\chi} \chi^{4}}{4}-\mu^{2} \chi^{2} V(\Phi)\right)},
$$

where $m_{P l} \approx 1.2 \cdot 10^{19} \mathrm{GeV}$ is the Planck mass.

The interaction introduced above leads to the following scenario. During inflation the magnitude of the inflaton field $\Phi$ decreases and when it reaches vicinity of $\Phi_{0}$ two minima appear in the potential

$$
U(\Phi, \chi)=\left(\frac{1}{2} m^{2}-\mu^{2} V(\Phi)\right) \chi^{2}+\frac{1}{4} \lambda_{\chi} \chi^{4}+\frac{1}{2} M^{2} \Phi^{2}
$$

at constant $\Phi$, so the point $\chi=0$ becomes local maximum.

Therefore, in the spatial regions where the field $\chi$ turns out to be positive (due to fluctuations) it rolls down to the positive minimum $\eta>0$, and in the regions where $\chi$ is negative it goes to the negative minimum $-\eta<0$. Thus, due to initial fluctuations we should obtain domains of the field $\chi$ which will provide the source of $C P$ violation, so in the domains with positive vacuum expectation value $\langle\chi\rangle$ we will have domain of matter while in the domain with negative $\langle\chi\rangle$ we will have domain of antimatter. After the interaction term $V(\Phi)$ is switched off there is again single minimum $\chi=0$ so there is no domain walls problem at the present time.

We expect that the distances between domains, as well as domain sizes exponentially grow with the scale factor $a(t)$ (see Section 3 for related problem consideration), so at the present time these domains are of cosmological size and they are separated by large distance which prevents the annihilation at their boundaries.

In [14] what happens at different stages of the scenario was considered, so it is possible to put the limits on initial conditions and parameters of the model. We will omit it here and we will check that the described scenario is indeed operative by performing the numerical calculations in the homogeneous case with the following set of parameters in those limits (all dimensional values are given in the Planck mass, $m_{P l}$, units):

$$
\Phi_{i n}=4, \Phi_{0}=3.1, \Phi_{1}=0.02, M=10^{-6}, \chi_{i n}=10^{-6}, m=10^{-10}, \lambda_{\chi}=2 \cdot 10^{-3}, \mu=10^{-4} .
$$

In Figs. 1 and 2 the results of numerical simulation are shown. One can see that the interaction of the inflaton field $\Phi$ with the field $\chi$ does not break the standard inflation, $\Phi(t)$ only slightly deviates from straight line around $\Phi=\Phi_{0}=3.1 \mathrm{~m}_{P l}$.

In Fig. 2 one can see how the field $\chi$ rolls down to the minimum of the potential $\eta(t)>0$ and oscillates there. Then it goes back to the old minimum at $\chi=0$, but even at the end of inflation $\chi$ 

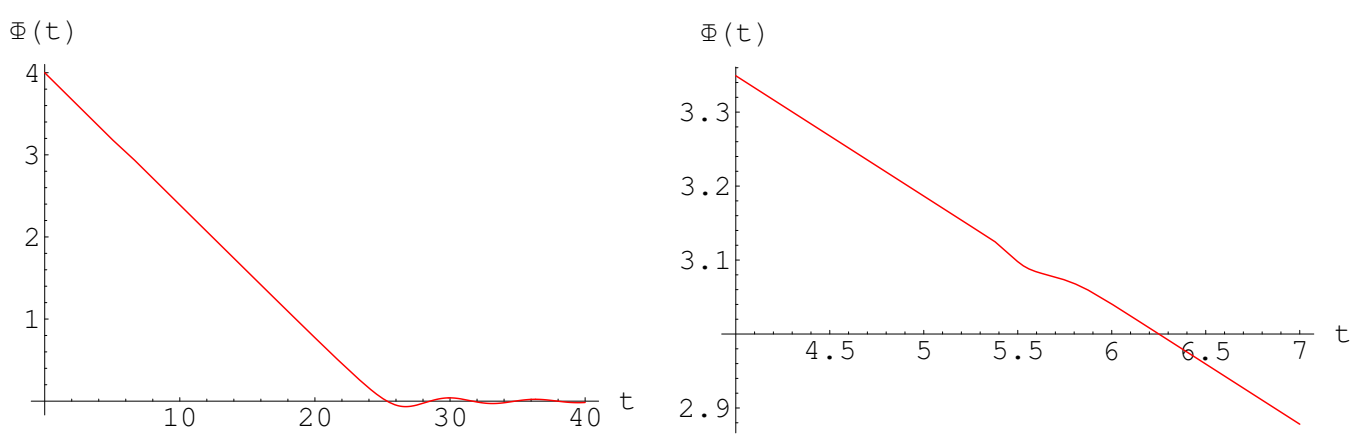

Figure 1: Time evolution of the inflaton field $\Phi(t)$ for the parameters (9). Time $t$ is measured in units of $M^{-1}$ and $\Phi$ is measured in units of $m_{P l}$. The right plot shows the evolution of $\Phi$ in more detail near the point $\Phi=\Phi_{0}=3.1 \mathrm{~m}_{P l}$.
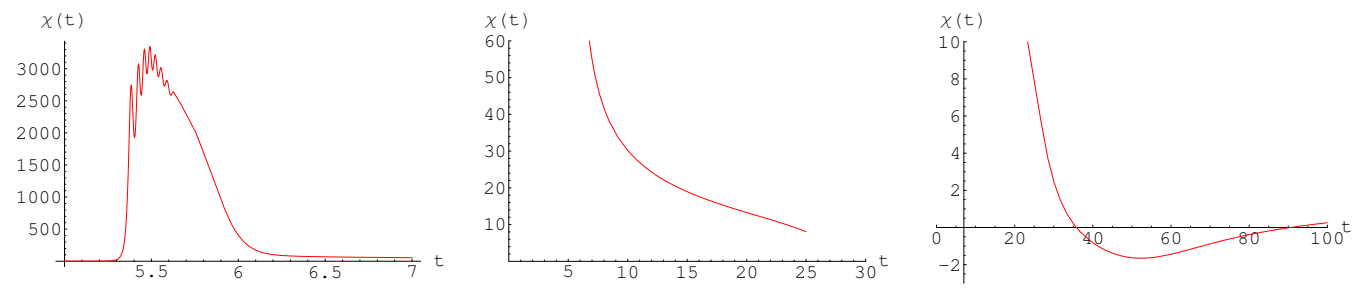

Figure 2: Time dependence of the field $\chi(t)$ for the parameters (9). Time $t$ is measured in units of $M^{-1}$ and $\chi$ is measured in units of $M$. All the three plots depict the evolution of $\chi$ during different time intervals. In the middle and right plots one can see that at the end of inflation, $t \approx 25 M^{-1}$, the field $\chi$ remains quite large.

remains an order of magnitude larger than it was at the beginning $\left(\chi \sim 10 M\right.$ at $t \approx 25 M^{-1}$, while $\chi_{\text {in }}=1 M$ ). At the moment $t \approx 35 M^{-1}$ the field $\chi$ crosses zero and starts slow oscillations with very low frequency, which could be much lower than the characteristic rate of baryogenesis.

So we see that parameters (9) are chosen in a proper way.

\subsection{Production of heavy particles after inflation and baryogenesis}

In the suggested model the $C P$-odd scalar field $\chi$ provides the source of $C P$ violation for one of the BAU generation mechanisms. Here we provide the example of how it can be achieved.

As it is commonly known, the stage of inflation is followed by the stage of (re)heating, during which the very heavy $X$ particles can be produced through decay of inflaton field (see e.g. book [16]) or from vacuum fluctuations in gravitational field [17].

Let us consider the situation when the inflaton field $\Phi$ interacts with $X$ particles (for example, scalar bosons) through the coupling $g_{X}^{2} \Phi^{2} X^{2}$. As is shown in [18-20], the particle production can be strongly enhanced due to parametric resonance. For an unsuppressed production it is essential that the resonance is broad, which is true for the wide range of coupling constant, $g_{X} \gg 4 \cdot 10^{-6}$. Because of resonance, very heavy $X$-bosons with masses even of GUT scale $m_{X} \sim\left(M m_{P l}\right)^{1 / 2} \sim 10^{16} \mathrm{GeV}$ can 
be produced under quite reasonable assumption $g_{X} \sim 1$ [16]. Moreover in this case of comparatively large coupling constant $g_{X} \sim 1$ the decay of the inflaton field occurs very rapidly.

Let us assume that the produced $X$-bosons in turn decay into fermions, for example, into quarkquark and antiquark-antilepton pairs, $X \rightarrow q q$ and $X \rightarrow \bar{q} \bar{l}$, respectively. If the corresponding coupling constants are large enough, the $X$-bosons decay very quickly. Therefore, the field $\chi(t)$ may remain non-zero yet to the moment when $X$-bosons have been completely decayed, indeed $\chi \sim 2 M \sim 10^{13} \mathrm{GeV}$ at $t=30 M^{-1}$ (see Fig. 2).

To provide the source of $C P$ violation for these decays we suppose that $\chi$ interacts with the produced fermions as

$$
L_{\chi \psi \psi}=g_{k l} \chi \bar{\psi}^{k} i \gamma_{5} \psi^{l}=i g_{k l} \chi\left(\bar{\psi}_{L}^{k} \psi_{R}^{l}-\bar{\psi}_{R}^{k} \psi_{L}^{l}\right),
$$

where $k$ and $l$ denote the fermion flavor (sum over repetitive indices is assumed), $\psi_{R}=\left(\left(1+\gamma_{5}\right) / 2\right) \psi$, $\psi_{L}=\left(\left(1-\gamma_{5}\right) / 2\right) \psi$. Since $\chi$ is supposed to be electrically neutral, it interacts with quarks with the same electric charge, so $k$ and $l$ either run over $u, c, t$ or $d, s, b$ and there are no cross terms.

The interaction of fermions with pseudoscalar field $\chi$ can be "rotated away" two unitary transformations $\psi_{R} \rightarrow \psi_{R}^{\prime}=U_{R} \psi_{R}$ and $\psi_{L} \rightarrow \psi_{L}^{\prime}=U_{L} \psi_{L}$. However, the interaction of fermions with vector (gauge) boson $X$ remains the same under these transformations:

$$
g_{R k l} X_{\mu} \bar{\psi}_{R}^{k} \gamma^{\mu} \psi_{R}^{l}+g_{L k l} X_{\mu} \bar{\psi}_{L}^{k} \gamma^{\mu} \psi_{L}^{l} \rightarrow g_{R a b}^{\prime} X_{\mu} \bar{\psi}_{R}^{a} \gamma^{\mu} \psi_{R}^{b}+g_{L a b}^{\prime} X_{\mu} \bar{\psi}_{L}^{a} \gamma^{\mu} \psi_{L}^{b}
$$

here $g_{R}^{\prime}=U_{R} g_{R} U_{R}^{\dagger}$ and $g_{L}^{\prime}=U_{L} g_{L} U_{L}^{\dagger}$ are matrices of coupling constants in mass eigenstate basis, so $\psi^{a}$ describes $a$-th sort of fermion with definite mass. The constants $g_{a b}^{\prime}$ are complex in general case, and if there are at least three species of fermions, one cannot rotate away simultaneously all phases in complex matrices $g_{R, L}^{\prime}[21]$. The complexity of the coupling constants means that $C P$ is violated in the $X$-boson decays [22]. The magnitude of this $C P$ violation depends on the value of the field $\chi$ through the matrices $U_{R, L}$ and coupling constants $g_{R, L}^{\prime}$. Since $\chi$ is essentially non-zero after the end of inflation and during baryogenesis, the $C P$-odd effects can be large enough.

We assume also that gauge interactions involve fermions with certain chirality, see (11), and thus these interactions break $C$-invariance.

$C$ and $C P$ violation is one of the necessary Sakharov conditions of generation of baryon asymmetry [23]. The another one is baryon number violation, so one needs to assume also that in the decays of $X$-bosons the baryon number is not conserved. Let $\delta$ be the baryon asymmetry generated in the decay of one $X$-boson. Then it can be easily demonstrated [16] that the ratio of the baryon number density to the entropy density is estimated as

$$
\Delta_{B}=\frac{n_{B}-n_{\bar{B}}}{s} \sim \delta \frac{h}{g_{X}}\left(\frac{m_{t h}}{m_{P l}}\right)^{1 / 2},
$$

where $h$ and $g_{X}$ are typical coupling constants of $X$-boson with fermions and inflaton, respectively, $m_{t h}$ is mass scale of the theory. It is quite reasonable to believe that $h / g_{X} \sim 1$ and $m_{t h} \sim M \sim 10^{-6} m_{P l}$, so one has $\Delta_{B} \sim 10^{-3} \delta$. Thus, to get observed value $\Delta_{B} \simeq 0.86 \cdot 10^{-10}$ it is sufficient to have only $\delta \sim 10^{-7}$. Such small $\delta$ seems to be easily produced in the decay of $X$-boson. Therefore, the observed baryon asymmetry of the universe can be generated in the decays of $X$-bosons, without fine tuning of parameters of the theory. 


\section{Domain walls in expanding universe}

\subsection{Stationary solutions}

In spatially flat section of de Sitter universe the expansion rate, $H=\dot{a} / a$, is constant and the scale factor evolves as $a(t)=\exp H t$. The FLRW metric for such universe has the form:

$$
d s^{2}=d t^{2}-e^{2 H t}\left(d x^{2}+d y^{2}+d z^{2}\right)
$$

Let us consider a model of real scalar field $\varphi$ with the Lagrangian

$$
\mathcal{L}=\frac{1}{2} g^{\mu \nu} \partial_{\mu} \varphi \partial_{\nu} \varphi-\frac{\lambda}{2}\left(\varphi^{2}-\eta^{2}\right)^{2}
$$

The corresponding equation of motion is

$$
\frac{1}{\sqrt{-g}} \partial_{\mu}\left(\sqrt{-g} g^{\mu \nu} \partial_{\nu} \varphi\right)=-2 \lambda \varphi\left(\varphi^{2}-\eta^{2}\right)
$$

In flat space-time, $H=0$, and in one-dimensional static case, $\varphi=\varphi(z)$, the equation takes the form $\frac{d^{2} \varphi}{d z^{2}}=2 \lambda \varphi\left(\varphi^{2}-\eta^{2}\right)$ and has a kink-type solution, which describes a static infinite domain wall. Without loss of generality we can assume that the wall is situated at $z=0$ in $x y$-plane:

$$
\varphi(z)=\eta \tanh \frac{z}{\delta_{0}}
$$

where $\delta_{0}=1 /(\sqrt{\lambda} \eta)$ has the meaning of the wall thickness (subscript 0 indicates that $H=0$ ).

Now let us consider an expanding universe with constant $H>0$. In this case, if one looks for stationary solution (see [12]), it is reasonable to suggest that the field $\varphi$ depends only on $z a(t)=$ $z \exp H t$, which is the proper distance from the wall. So, one can choose the following ansatz for $\varphi$ :

$$
\varphi=\eta \cdot f(u), \quad \text { where } u=H z e^{H t}
$$

where $u$ and $f$ are dimensionless. Then the equation of motion takes the form:

$$
\left(1-u^{2}\right) f^{\prime \prime}-4 u f^{\prime}=-2 C f\left(1-f^{2}\right)
$$

Here prime means the derivative with respect to $u$. It is noteworthy that all parameters of the problem are combined into a single positive constant $C=1 /\left(H \delta_{0}\right)^{2}=\lambda \eta^{2} / H^{2}>0$.

Since we are interested in kink-type solutions, the boundary conditions should be

$$
f(0)=0, \quad f( \pm \infty)= \pm 1 .
$$

Corresponding numerical solutions for different values of parameter $C$ are shown in Fig. 1 of $[12,15]$. We see that the larger is $C$ the closer is the solution to the flat space-time one $(H=0)$, as is naturally expected.

As it is noticed in ref. [12] the stationary solutions can be found only for $C>2$, but no explanation of this observation is given therein. For the detailed explanation see [15]. 


\subsection{Evolution of domain walls beyond the stationary limit}

As we have seen in the Section 3.1, Eq. (18) allows to find the field configurations, which describe stationary domain walls in expanding universe. However, it is also interesting to see how domain walls evolve from some initial states. Beyond the stationary approximation we can find not only solution for $C>2$ but also for $C \leq 2$, for which the stationary approximation does not exist.

To this end one should solve the original equation of motion (15) in the case when the field $\varphi$ is a function of two independent variables, $z$ and $t$. It is convenient to introduce dimensionless variables $\tau=H t, \zeta=H z$ and function $f(\zeta, \tau)=\varphi(z, t) / \eta$. As a result one obtains the equation

$$
\frac{\partial^{2} f}{\partial \tau^{2}}+3 \frac{\partial f}{\partial \tau}-e^{-2 \tau} \frac{\partial^{2} f}{\partial \zeta^{2}}=2 C f\left(1-f^{2}\right),
$$

where $C=\lambda \eta^{2} / H^{2}=1 /\left(H \delta_{0}\right)^{2}>0$ as it was above.

The boundary conditions for the kink-type solution should be

$$
f(0, \tau)=0, \quad f( \pm \infty, \tau)= \pm 1
$$

and we choose the initial configuration as the domain wall with "natural" thickness $1 / \sqrt{C}$ (with respect to dimensionless coordinate $\zeta$ ) and zero time derivative.

The evolution of the domain wall for $C=4$ is depicted in Figs. 3a-3b. The stationary solution is shown by black curve (it is denoted by "BV" because of Basu and Vilenkin who found it [12]). One sees that the domain wall evolves in somewhat non-trivial way. At the very beginning the wall starts to broaden in terms of the proper distance from the wall, $z H \operatorname{exp~} H t$, and at some moment it becomes wider than the stationary solution. However, afterwards the wall broadening changes to contraction. Finally, the wall comes to the stationary configuration after several damped oscillations around it.

Let us consider now smaller values of parameter $C$. When it is close to its critical value $C=2$, the stationary domain wall is quite wide. Therefore, if the initial thickness of the wall is $1 / \sqrt{C}$, one should expect that the non-stationary solution approaches to the stationary one only after quite long time. The corresponding evolution of domain wall for $C=2.5$ is depicted in Figs. 3c-3d.

When $C$ is very close to 2, the solution of Eq. (20) converges to the stationary one very slowly. One can see that in Fig. 3e-3f for $C=2.05$. In Fig. 3f we chose initial thickness to be $4 / \sqrt{C}$ so it takes less time for the solution to approach the stationary one.

It may be also interesting to choose an initial domain wall with the thickness greater than that of the stationary solution. For such case see Fig. 4, where the evolution of domain wall with initial thickness $3 / \sqrt{C}$ for $C=2.5$ is presented. The wall also eventually comes to the stationary configuration.

For $C \leq 2$ there are no stationary solutions at all. Evolution of domain wall for such values of parameter $C$ is shown in Fig. 5a-5b. One can see that the domain wall thickness increases indeed.

In Section 2 we assumed that the size of the transition regions between baryon and antibaryon domains (such regions formed in the place of the disappeared domain walls) could be exponentially large, so the domains would be separated by a few Mpc (in the present day scale). Let us check now if this can be true. So we have to calculate how fast the domain wall thickness can increase in de Sitter universe. In our model the wall is described at the initial moment by hyperbolic tangent $\varphi / \eta=\tanh (z / \delta)$, where the wall thickness is denoted by $\delta$. Although in the realistic course of the evolution the wall is not "pure" hyperbolic tangent, nevertheless one can use the same definition for the thickness $\delta(t)$ as the value of the coordinate $z$ at the position where the field $\phi$ reaches the value $\phi / \eta=\tanh 1 \approx 0.76$. 


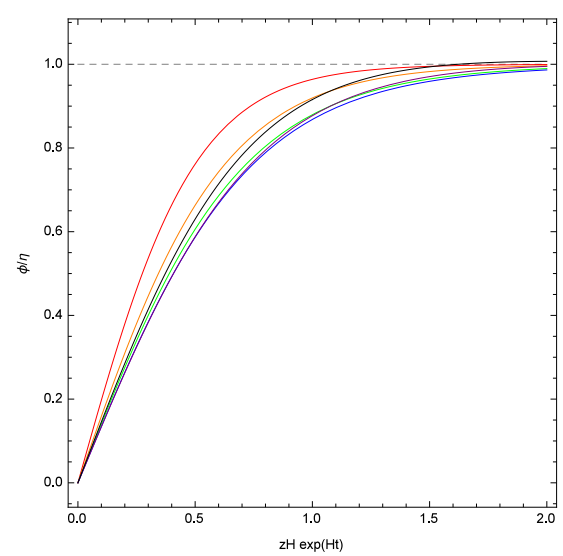

(a) $C=4.0$, IC: $f(\zeta, 0)=\tanh \sqrt{C} \zeta$.

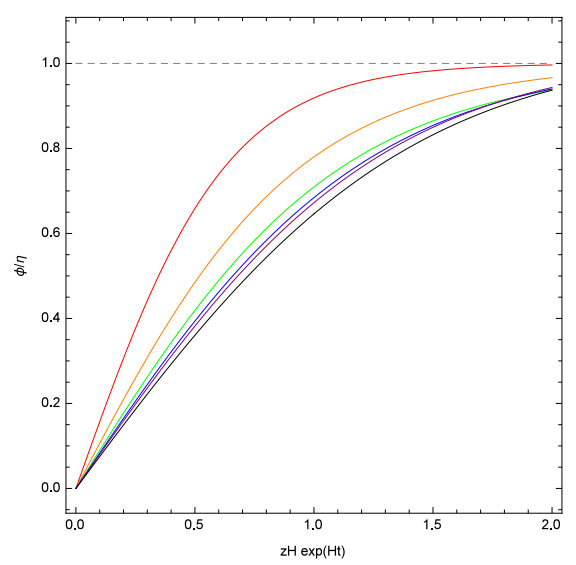

(c) $C=2.5$, IC: $f(\zeta, 0)=\tanh \sqrt{C} \zeta$.

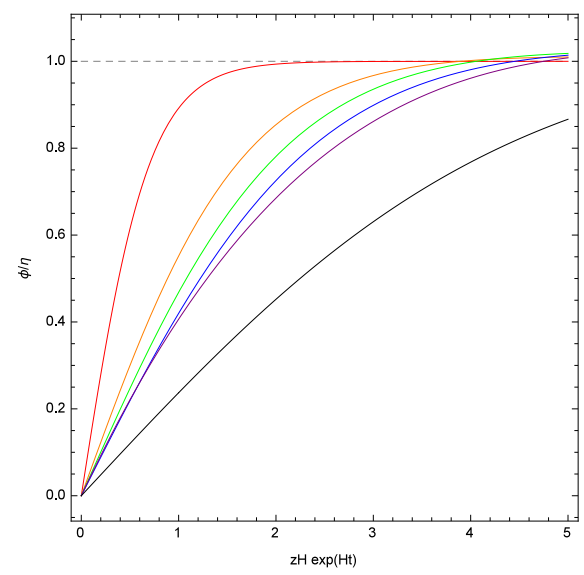

(e) $C=2.05$, IC: $f(\zeta, 0)=\tanh (\sqrt{C} \zeta)$.

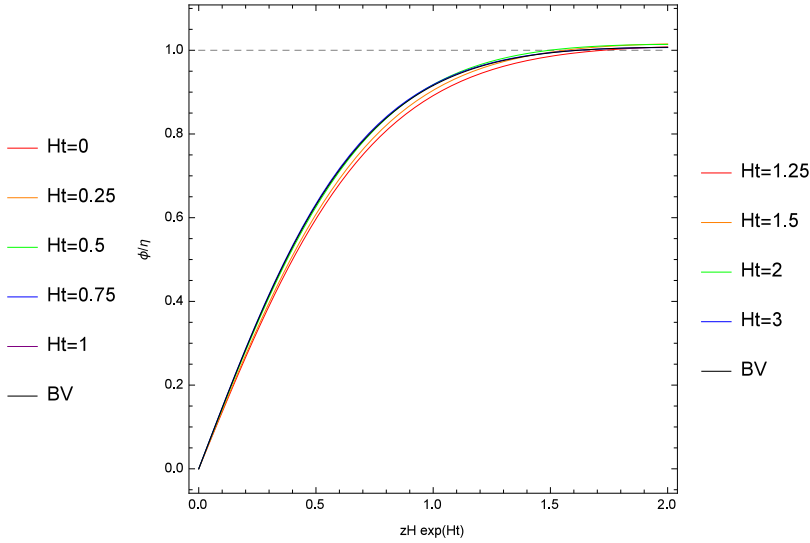

(b) $C=4.0$, IC: $f(\zeta, 0)=\tanh \sqrt{C} \zeta$.

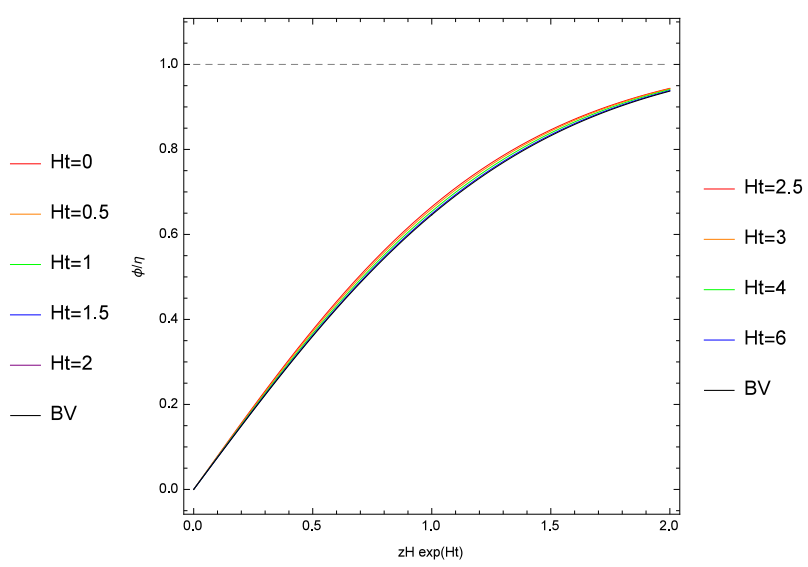

(d) $C=2.5$, IC: $f(\zeta, 0)=\tanh \sqrt{C} \zeta$.
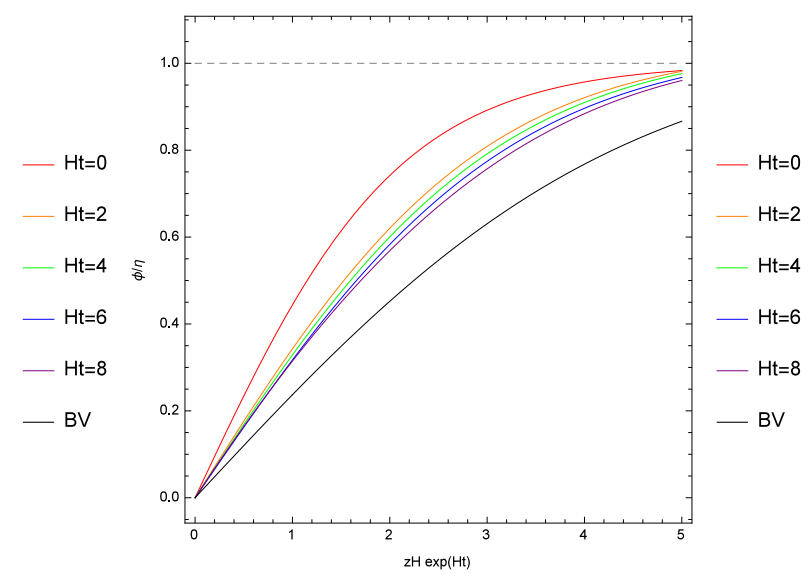

(f) $C=2.05$, IC: $f(\zeta, 0)=\tanh (\sqrt{C} \zeta / 4)$.

Figure 3: Domain wall evolution for values of $C>2$ with corresponding initial configurations (IC). Black curve corresponds to stationary solution. 


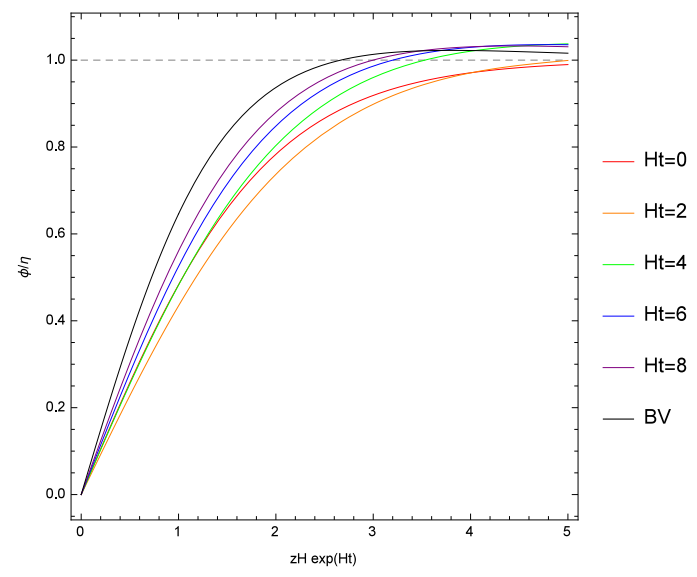

Figure 4: Evolution of domain wall for $C=2.5$. Initial configuration is $f(\zeta, 0)=\tanh (\sqrt{C} \zeta / 3)$.

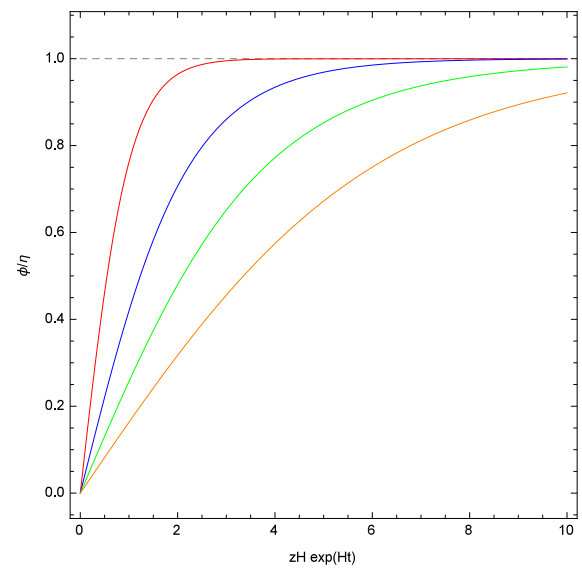

(a) $C=1.0$.

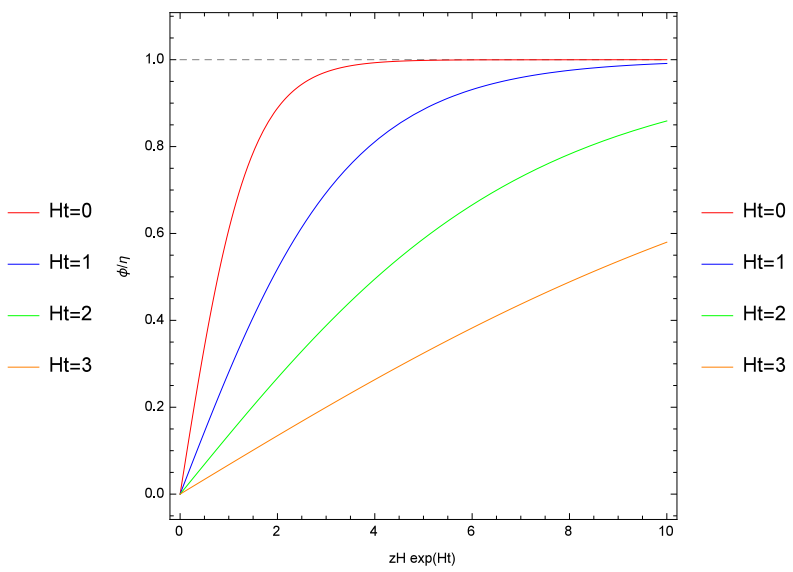

(b) $C=0.5$.

Figure 5: Domain wall evolution for values of $C<2$. Initial configuration is $f(\zeta, 0)=\tanh (\sqrt{C} \zeta)$.

In Fig. 6 the time dependence of the physical width of the wall for different values of $C$ is presented. In the left plot $(C>2)$ one can see that all the curves indeed tend to constant values corresponding to stationary solutions (dashed lines). Oscillatory behavior mentioned above is also apparent. Right plot contains curves for $C<2$. It is clear that for $C=1$, i.e. for not very small values of $C$, the wall thickness increases slower than exponent. However, for smaller values of $C$, e.g. $C \lesssim 0.1$ the rate of the wall expansion is the exponential one with a good accuracy.

The Hubble constant, $H$, plays the role of friction in this problem and when it dominates over the potential term $(C \ll 1)$, the field configuration as a function of coordinates becomes almost static. Therefore the width of the wall is growing nearly as the scale factor $a(t)$, i.e. almost exponentially. This is why there are no stable solutions for $C \ll 1$. 


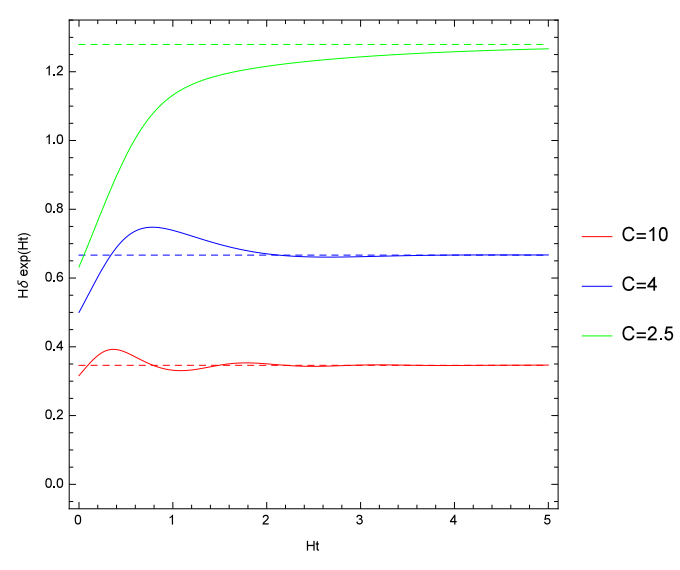

(a) $C>2$.

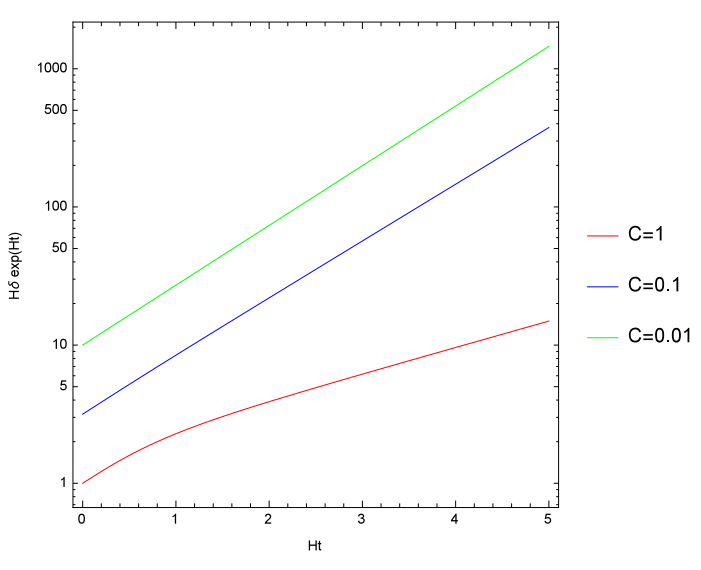

(b) $C<2$.

Figure 6: Time dependence of domain wall thickness. Dashed lines describe the stationary solutions.

\section{Conclusions}

The suggested model of baryogenesis may lead to baryo-symmetric universe with cosmologically large domains of matter and antimatter, avoiding the domain wall problem. Breaking of charge symmetry is due to a scalar field $\chi$, which is not completely relaxed down to equilibrium by the end of the baryogenesis. In classification of different types of $C P$ violation which might be operative in cosmology this type is called dynamical one [24]. Inflation is an essential ingredient of the scenario since the distance between the domains and their sizes should be cosmologically large.

This scenario inspired the consideration of the related problem: time evolution of thick domain walls in a de Sitter universe. We have shown that for large values of parameter $C>2$ the initial kink configuration in a de Sitter background tends to the stationary solution obtained by Basu and Vilenkin [12]. For $C<2$ the stationary solution does not exist and the width of the wall infinitely grows with time. For $C \lesssim 0.1$ the rate is close to the exponential one within the precision of our numerical calculations. This result is essential for application of spontaneous breaking of symmetry between particles and antiparticles to realistic cosmology.

We acknowledge support of the RSF Grant No. 16-12-10037. SG is also supported under the grants RFBR No. 16-32-60115, 16-32-00241, 16-02-00342. In addition, SG is grateful to Dynasty Foundation for support.

\section{References}

[1] A.D. Dolgov, Phys. Rept. 222, 309 (1992)

[2] A.D. Dolgov, Surv. High Energ. Phys. 13, 83 (1998)

[3] V.A. Rubakov and M.E. Shaposhnikov, Phys. Usp. 39, 461 (1996)

[4] A. Riotto and M. Trodden, Ann. Rev. Nucl. Part. Sci. 49, 35 (1999)

[5] M. Dine and A. Kusenko, Rev. Mod. Phys. 76, 1 (2003)

[6] A.G. Cohen, A. De Rujula and S.L. Glashow, Astrophys. J. 495, 539 (1998)

[7] Ya. B. Zeldovich, I.Yu. Kobzarev and L.B. Okun, Sov. Phys. JETP 40, 1 (1974) 
[8] V.A. Kuzmin, I.I. Tkachev and M.E. Shaposhnikov, Pisma Zh. Eksp. Teor. Fiz. 33, 557 (1981)

[9] V.A. Kuzmin, M.E. Shaposhnikov and I.I. Tkachev, Phys. Lett. B 105, 159 (1981)

[10] V.A. Kuzmin, M.E. Shaposhnikov and I.I. Tkachev, Phys. Lett. B 105, 167 (1981)

[11] V.A. Kuzmin, M.E. Shaposhnikov and I.I. Tkachev, Nucl. Phys. B 196, 29 (1982) [Erratum ibid. B 202, 543 (1982)]

[12] R. Basu and A. Vilenkin, Phys. Rev. D 50, 7150 (1994)

[13] N.A. Voronov, A.L. Dyshko and N.B. Konyukhova, Phys. Atom. Nucl. 68, 1218 (2005)

[14] A.D. Dolgov, S.I. Godunov, A.S. Rudenko and I.I. Tkachev, JCAP 10, 027 (2015)

[15] A.D. Dolgov, S.I. Godunov, A.S. Rudenko, JCAP 10, 026 (2016).

[16] D.S. Gorbunov, V.A. Rubakov, Introduction to the theory of the early universe: Cosmological perturbations and inflationary theory, World Scientific (2011).

[17] V.A. Kuzmin and I.I. Tkachev, Phys. Rev. D 59, 123006 (1999)

[18] Yu.V. Shtanov, J.H. Traschen and R.H. Brandenberger, Phys. Rev. D 51, 5438 (1995)

[19] L. Kofman, A.D. Linde and A.A. Starobinsky, Phys. Rev. Lett. 73, 3195 (1994)

[20] L. Kofman, A.D. Linde and A.A. Starobinsky, Phys. Rev. D 56, 3258 (1997)

[21] M. Kobayashi and T. Maskawa, Prog. Theor. Phys. 49, 652 (1973)

[22] D.V. Nanopoulos and S. Weinberg, Phys. Rev. D 20, 2484 (1979)

[23] A.D. Sakharov, JETP Lett. 5, 24 (1967); Sov. Phys. Usp. 34 (1991) 392

[24] A.D. Dolgov, CP violation in cosmology, in the proceedings of 163rd Course of International School of Physics 'Enrico Fermi': CP Violation: From Quarks to Leptons, Varenna, Italy, July 19-29, 2005 [hep-ph/0511213]. 